\title{
Thermostat Device
}

National Cancer Institute

\section{Source}

National Cancer Institute. Thermostat Device. NCI Thesaurus. Code C50221.

A device designed to regulate temperature by controlling the starting and stopping of a heating/cooling system. 